Original Article

\title{
Complex DECAF scores prolong the hospital stay of patients suffering from COPD Mobin Ahmed', Sheeba Faryal ${ }^{2}$ \& Khalid Sheikh ${ }^{2}$
}

1. Asian Institute of Medical Sciences (AIMS)

2. Liaquat University of Medical \& Health Sciences (LUMHS)

Corresponding Author: dr.mubeen41@gmail.com

\begin{abstract}
Objective Patients with Complex the Dyspnoea, Eosinopenia, Consolidation, Acidaemia, and atrial Fibrillation (DECAF) scores prolong the hospital stay of patients suffering from Chronic Obstructive Pulmonary Disease (COPD). Method Patients admitted to respiratory wards in tertiary care hospital, Baluchistan with COPD between June 2016 to September, 2015 were prospectively reviewed and DECAF score applied to each patient. Duration of hospitalization was then correlated with total DECAF scores. Result Out of 118 total admissions, 103 were reviewed as 15 patients died. $71 \%$ were male, mean age was 69.6 years and average of 15 days of hospitalization. The duration was longest in those with DECAF scores of 3-5 and lowest in those with scores of 01. Conclusion The DECAF Score is a simple and effective clinical tool that can risk stratify hospitalized patients with COPD and could therefore help clinicians managing this fatal condition.
\end{abstract}

\section{Keywords}

DECAF, COPD, Chronic Obstructive Pulmonary Disease, Dyspnoea, Eosinopenia, Consolidation, Acidaemia, atrial Fibrillation

\section{Introduction}

More than 2,410 million globally suffers from COPD with Pakistan sharing the burden of 6.9 million people. COPD is one of the biggest threat to health and set to become the 4th leading cause of death by $2030^{1}$. Cigarette smoking is the most commonly encountered risk factor of COPD disease found in Pakistan. Characterised by persistent airflow limitation associated with an enhanced chronic inflammatory response in the airways and the lung to noxious particles or gases. Clinical features include shortness of breath, persistent cough, coughing up mucus, wheezing, fatigue/tiredness, weight loss, morning headaches are alarming signs of the disease. In Pakistan, it is more prevalent in men as compared to women and prevalence of COPD increases with age, primarily affects people of 40 years and above $^{2}$. The challenges in COPD management are access, cost, and non-adherence/poor compliance. Access to medications for chronic disease management is limited in many low and middle-income countries, resulting in substandard care and preventable morbidity and mortality. Hospitalisation due to serious exacerbations of COPD is common, and subsequent mortality high ${ }^{3,4}$. The DECAF score was derived for accurate prediction of mortality and risk stratification to inform patient care. Severe exacerbations of COPD account for one in eight hospital admissions and are associated with worsening symptoms, lung function, healthrelated quality of life, and mortality risk ${ }^{4}$. Usually Clinicians are unable accurately to predict prognosis in patients hospitalised with acute COPD because of that a robust prediction tool, which stratifies patients according to mortality risk is always a need 
that can help in identifying low-risk groups, and early escalation or appropriate palliation for high-risk groups. The Dyspnoea, Eosinopenia, Consolidation, Acidaemia, and atrial Fibrillation (DECAF) score was derived in a large cohort of consecutive patients hospitalised with COPD, is simple to apply at the bedside and predicts in hospital mortality using indices routinely available on admission $^{5}$. The score comprises five predictors, the strongest of which is stable state dyspnoea, as measured by the extended Medical Research Council Dyspnoea score ${ }^{6}$. The major objective behind this study was to explore the significance of using DECAF scoring in patients suffering from COPD in unexplored populace of Baluchistan as well as to identify the relation of higher DECAF scores with duration of hospitalization.

\section{Methodology}

This study was carried out at the Chest \& Department from November 2015 to April 2016. It included 118 patients with a mean age of $69.6 \pm 7$; Out of 118 total admissions, 103 were reviewed as 15 patients died. $71 \%$ were male, mean age was years and average of 15 days of hospitalization. The duration was longest in those with DECAF scores of 3-5 and lowest in those with scores of 0-1. All studied patients were subjected Thorough medical history, clinical examination, Plain Chest X- ray, Routine laboratory investigations, Assessment of the DECAF Score according to Steer et al. Statistical analysis was performed with the SPSS statistical software package version 20 (SPSS Inc., Chicago, L, USA). P-value $<0.05$ was considered significant.

\section{Result}

The results shows the socio-demographic data of all studied patients with the presence of cerebrovascular disease, atrial fibrillation and renal comorbidity. The data shows the average serum levels of potassium, urea, creatinine, glucose, CRP and albumin. Also, hemoglobin level and eosinophil that showed altered values.

Table 1 DECAF Score.

\begin{tabular}{ll}
\hline Variable & Score \\
\hline Dyspnoea & \\
eMRCD 5a & 1 \\
eMRCD $5 b$ & 2 \\
Eosinopenia $\left(<0.05 \times 10^{9} \wedge\right)$ & 1 \\
Consolidation & 1 \\
Acidaemia $(\mathrm{pH}<7.3)$ & 1 \\
Atrial fibrillation & 1 \\
Total DECAF Score & 6 \\
\hline
\end{tabular}

DECAF, Dyspnoea, Eosinopenia, Consolidation, Acidaemia and atrial Fibrillation; eMRCD, extended MRC dyspnoea.

Table 2 Clinical and Radiological Findings at Admission.

\begin{tabular}{ll}
\hline Purulent sputum & $60 \%$
\end{tabular}

\begin{tabular}{ll}
\hline Ineffective cough & $11.4 \%$ \\
\hline Lower limb edema & $25.7 \%$
\end{tabular}

Acute confusion $\quad 10.3 \%$

Herat rate/min $\quad 100.3 \pm 21.2$

Systolic blood pressure $140 \pm 28$
(mm Hg)

Diastolic blood
pressure $(\mathbf{m m ~ H g})$

Respiratory rate/min $24.5 \pm 5.7$ 
Table 3 Laboratory Investigations.

\begin{tabular}{ll}
\hline Parameter & $\begin{array}{l}\text { Survivors } \\
(\mathbf{n o}=\mathbf{1 7 5})\end{array}$ \\
\hline Sodium (mEq/l) & $136.4 \pm 4.6$ \\
\hline Potassium (mEq/l) & $4.3 \pm 0.5$ \\
\hline Chloride (mEq/l) & $98.6 \pm 8.9$ \\
\hline Urea (mmol/l) & $6.5 \pm 2.1$ \\
\hline Creatinine (mg/dl) & $0.92 \pm 0.21$ \\
\hline Albumin (g/dl) & $3.9 \pm 0.45$ \\
\hline Glucose (mg/dl) & $126 \pm 18$ \\
\hline Hemoglobin $(\mathbf{g} / \mathbf{d l})$ & $13.8 \pm 1.8$ \\
\hline CRP $(\mathbf{m g} / \mathbf{d l})$ & $5.6 \pm 3.6$ \\
\hline $\begin{array}{l}\text { White cell count } \\
(\cdot \mathbf{1 0 3} / \mathbf{d l})\end{array}$ & $11.9 \pm 3.6$ \\
\hline $\begin{array}{l}\text { Neutrophil count } \\
(\cdot \mathbf{1 0 3} / \mathbf{d l})\end{array}$ & $9.2 \pm 3.4$ \\
\hline $\begin{array}{l}\text { Esinophil count } \\
(\cdot \mathbf{1 0 3} / \mathbf{d l})\end{array}$ & $0.1 \pm 0.1$ \\
\hline $\begin{array}{l}\text { Cerebrovascular } \\
\text { disease }\end{array}$ & $11.6 \%$ \\
\hline $\begin{array}{l}\text { Ischemic heart } \\
\text { disease }\end{array}$ & $31 \%$ \\
\hline Hypertension & $37 \%$ \\
\hline Diabetes & $5.3 \%$ \\
\hline $\begin{array}{l}\text { Atrial fibrillation } \\
\text { Renal comorbidity }\end{array}$ & $7.6 \%$ \\
\hline
\end{tabular}

\section{Graph 1 showing hospitalization duration and DECAF Score}

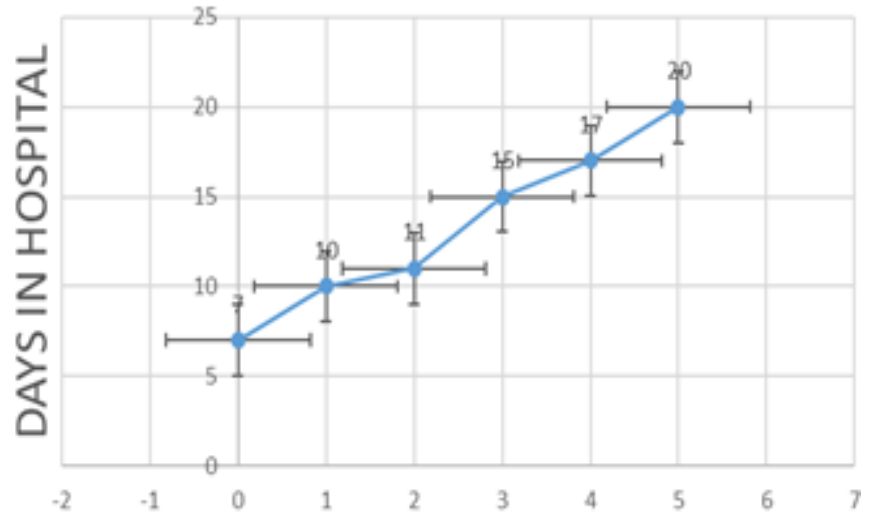

DECAF SCORE

\section{Discussion}

The changeability in mortality rates globally as well as locally for patients with COPD admitted for critical respiratory failure suggests that significant heterogeneity exists among populations ${ }^{7}$. The differences in patient physiognomies, quality of care as well as ecological account is of much variability. There is relatively less amount of literature available to make these considerations more factual. Recognizing upon hospitalization those at higher risk of developing complications during their hospital stay and could be useful for determining the appropriate level of care, pivotal for recognition of effective therapies, and timely discharges. As DECAF Score is believed to accurately predict in hospital mortality for patients with $\mathrm{COPD}^{8}$. The DECAF Score can be used as a simple prognostic tool, incorporating clinical and laboratory information available routinely on admission in patients hospitalized. Most of the predictors associated with higher complications incidence and prolong hospitalization (Graph:1) are consistent with previously published studies in COPD like esinopenia was associated with a higher in hospital mortality in AECOPD $^{9,10}$, but the study population was small $(n=118)$. Our results show that esinopenia and may be a useful marker of sepsis in patients who are receiving intensive care. The strong prognostic effect of eosinopenia may reflect the severity of the accompanying acute inflammatory response ${ }^{11}$. The DECAF Scores in present study shows promise for the risk stratification of patients hospitalized for each grade of the DECAF Score with risk categories. Our Findings suggest that a high DECAF Score $(\geqslant 3)$ might be used as a guide to early escalation of care. 


\section{Conclusion}

Duration of hospitalization depends on several factors like physiological status, severity, co-morbidities and accessibility of health and communal care. Patients with high DECAF scores were generally more unwell and have longer recovery duration. Once suitable these patients should be discharged with help of early supported care teams.

\section{Conflict of Interest}

None.

\section{Acknowledgement}

None.

\section{References}

1. Bustamante-Fermosel, A., De MiguelYanes, J. M., Duffort-Falcó, M., \& Muñoz, J. (2007). Mortality-related factors after hospitalization for acute exacerbation of chronic obstructive pulmonary disease: the burden of clinical features. The American journal of emergency medicine, 25(5), 515-522.

2. Steer, J., Gibson, G. J., \& Bourke, S. C. (2010). Predicting outcomes following hospitalization for acute exacerbations of COPD. QJM: An International Journal of Medicine, 103(11), 817-829.

3. Steer, J., Norman, E. M., Afolabi, O. A., Gibson, G. J., \& Bourke, S. C. (2012). Dyspnoea severity and pneumonia as predictors of in-hospital mortality and early readmission in acute exacerbations of COPD. Thorax, 67(2), 117-121.

4. Nafae, R., Embarak, S., \& Gad, D. M. (2015). Value of the DECAF score in predicting hospital mortality in patients with acute exacerbation of chronic obstructive pulmonary disease admitted to Zagazig University Hospitals, Egypt. Egyptian Journal of Chest Diseases and Tuberculosis, 64(1), 35-40.
5. Steer, J., Gibson, J., \& Bourke, S. C. (2012). The DECAF Score: predicting hospital mortality in exacerbations of chronic obstructive pulmonary disease. Thorax, thoraxjnl-2012.

6. Son, J. H., Lee, J. Y., Yang, Y. M., Sung, W. Y., Seo, S. W., Kim, J. C., \& Lee, W. (2013). Utility of the DECAF Score in Patients Admitted to Emergency Department with Acute Exacerbation of Chronic Obstructive Pulmonary Disease. Korean Journal of Critical Care Medicine, 28(4).

7. Abidi, K., Khoudri, I., Belayachi, J., Madani, N., Zekraoui, A., Zeggwagh, A. A., \& Abouqal, R. (2008). Eosinopenia is a reliable marker of sepsis on admission to medical intensive care units. Critical Care, 12(2), R59.

8. World Health Organization, \& World Health Organization. Management of Substance Abuse Unit. (2014). Global status report on alcohol and health, 2014. World Health Organization.

9. N. Roche, M. Zureik, D. Soussan, et al, (2008). Predictors of outcomes in COPD exacerbation cases presenting to the emergency department, Eur. Respir. J. (32)953-961.

10. Ruiz-González, A., Lacasta, D., Ibarz, M., Martínez-Alonso, M., Falguera, M., \& Porcel, J. M. (2008). C-reactive protein and other predictors of poor outcome in patients hospitalized with exacerbations of chronic obstructive pulmonary disease. Respirology, 13(7), 1028-1033.

11. Patil, S. P., Krishnan, J. A., Lechtzin, N., \& Diette, G. B. (2003). In-hospital mortality following acute exacerbations of chronic obstructive pulmonary disease. Archives of internal medicine, 163(10), 1180-1186. 